\title{
Prescription de médicaments: sécuriser un processus complexe
}

- La prescription de médicaments est un processus complexe et une source potentielle d'erreur médicale. II est donc important de rendre le corps médical encore plus conscient de cette problématique.

- Les causes d'erreurs sont fréquemment liées au système de prescription. C'est la raison pour laquelle la FMH plaide pour une optimisation interdisciplinaire, interinstitutionnelle et pluridimensionnelle. Des analyses supplémentaires des causes sont également nécessaires, en particulier dans le domaine ambulatoire.

- De nombreuses erreurs de prescription sont évitables. Or, il existe déjà des outils adaptés à la pratique qui améliorent la sécurité des prescriptions; il faudrait donc les coordonner et les appliquer à large échelle.

- Une gestion transparente et constructive des erreurs de prescription doit être encouragée, car elle est la base des mesures d'amélioration concrètes.

Correspondance: FMH/DDQ Elfenstrasse 18 CH-3000 Berne 15 Tél. 0313591111 Fax 0313591112 ddq[at]fmh.ch

\section{Contexte}

Que ce soit dans le domaine ambulatoire ou hospitalier, la sécurité des médicaments est l'un des points les plus importants pour la sécurité des patients. C'est pourquoi, à l'instar d'autres pays et institutions telle l'OMS, la stratégie de la Confédération en matière de qualité dans le système de santé suisse met l'accent sur l'amélioration de la sécurité médicamenteuse. La prescription par le médecin joue un rôle central dans l'ensemble du processus thérapeutique médicamenteux, lequel est évidemment important, mais aussi particulièrement complexe et potentiellement risqué. En Suisse comme à l'étranger, il existe des approches prometteuses pour le sécuriser.

\section{L'avis de la FMH}

Fréquence des erreurs de prescription: des études nationales et internationales ont montré que les erreurs de prescription sont fréquentes, bien qu'il aurait été possible d'en éviter un grand nombre. Les patients sont les premiers touchés, mais les conséquences financières sont également importantes. La FMH est clairement d'avis que les erreurs de prescription sont beaucoup trop fréquentes. Outre les sources d'erreurs concrètes telles que les malentendus, la faible conscience du haut potentiel d'erreurs en est la cause essentielle. Afin d'accroître la sensibilité du corps médical aux erreurs de prescription, cette problématique devrait être encore mieux ancrée dans la formation pré- et postgraduée. Ce n'est qu'en augmentant la vigilance qu'il sera possible d'analyser les processus de prescription de manière précise et de les améliorer.

Causes des erreurs de prescription: la prescription de médicaments est un processus complexe et l'analyse des causes d'erreurs l'est tout autant. Ce ne sont souvent pas des facteurs isolés qui donnent lieu à des erreurs, mais un concours de circonstances. Dans le domaine ambulatoire en particulier, ces causes ont été trop peu analysées jusqu'ici. Il est nécessaire de procéder à de nouvelles études afin d'optimiser de manière ciblée la sécurité des prescriptions sur la base des données récoltées.

Gestion des erreurs de prescription: pour pouvoir analyser systématiquement les erreurs médicamenteuses, il importe de les gérer avec transparence, notamment par la mise en place d'un système d'annonce d'erreurs et par la gestion constructive de celles-ci. Cela permettra entre autres d'empêcher que des erreurs évitables ne se répètent. Etant donné qu'elles sont fréquemment liées au système de prescription, il sera utile de procéder à des analyses et à des discussions incluant tous les groupes professionnels concernés, par exemple au sein de cercles de qualité.

Amélioration de la sécurité des prescriptions: pour réduire les erreurs médicamenteuses, il faut impérativement améliorer la sécurité des prescriptions. Il existe d'ores et déjà de nombreux instruments de prévention avérés et prometteurs tels que les ordonnances informatisées ou les cercles de qualité interdisciplinaires entre médecins et pharmaciens. Ils doivent être soutenus et coordonnés pour permettre à partir de ces actions individuelles, le développement de mesures à large échelle. Il faudra également développer de nouveaux outils applicables à la pratique. Le groupe de travail eHealth de la FMH a élaboré des bases spécifiques et des prises de position quant au soutien électronique à l'établissement des ordonnances; il publiera ces documents sous peu.

Monitorage: afin d'améliorer durablement la sécurité des prescriptions, des mesures efficaces sont indispensables. Afin de vérifier si les mesures prises améliorent réellement la sécurité, il faudra mettre en place un monitorage régulier. On entend par là la réalisation d'études qui devraient permettre, à terme, de contrôler la fréquence des erreurs de prescription et d'analyser l'effet des mesures d'amélioration prises. 